Article

\title{
The Influence of Substrate Size Changes on the Coil Resistance of the Wireless Power Transfer System
}

\author{
Jiacheng $\mathrm{Li}^{1,2} \mathbb{C}$, Linlin Tan ${ }^{1,2, *}$, Xueliang Huang ${ }^{1,2}$, Ruoyin Wang ${ }^{1,2}$ and Ming Zhang ${ }^{1,2}$ \\ 1 Department of Electrical Engineering, Southeast University, No. 2 Sipailou, Nanjing 210096, China; \\ wldfy@foxmail.com (J.L.); xlhuang@seu.edu.cn (X.H.); ruoy_wang@seu.edu.cn (R.W.); \\ zhangming1212@seu.edu.cn (M.Z.) \\ 2 Key Laboratory of Smart Grid Technology and Equipment in Jiangsu Province, Nanjing 210096, China \\ * Correspondence: tanlinlin@seu.edu.cn; Tel.: +86-25-8379-4691 (ext. 815)
}

Received: 29 May 2020; Accepted: 18 June 2020; Published: 21 June 2020

\begin{abstract}
Wireless power transfer (WPT) technology has been widely used in many fields. Nevertheless, in the field of high power transmission, such as the WPT system of electric vehicles, the power transmission efficiency of WPT system lags behind that of wired charging due to losses brought by substrate shielding materials. In this regard, the conduction resistance of Litz-wire coils without substrate is analyzed first in this paper. Secondly, the induction resistance of the coil with single-layer and double-layer substrate materials is modeled. Then, through the establishment of a coil simulation and experimental platform with a single-layer substrate, a contrastive analysis of the variation trend of coil equivalent series resistance (coil ESR) at changing thickness and area and constant volume of the substrate is carried out in combination with the theory. The variation law of coil ESR at changing thickness and area and constant volume of double-layer substrate is also explored at the end of this paper. This is expected to contribute to the reduction of coil losses in the WPT system through a systematic study of the influence of substrate size changes on the coil resistance of the WPT system.
\end{abstract}

Keywords: wireless power transfer; coil resistance; substrate size

\section{Introduction}

The wireless power transfer (WPT) technology uses energy carriers (e.g., magnetic field, electric field, electromagnetic wave, etc.) in the physical space and realizes the transfer of electric energy from the power supply side to the load side based on a no-wire contacting mode $[1,2]$. This technology provides effective solutions and measures for reliable power supply of inspection robots [3], electric vehicles (EVs) [4], mobile phones [5], unmanned aerial vehicles [6], on-line EVs [7] and industrial application in high-voltage transmission networks [8], and avoids contact sparks, plug wear $[9,10]$, and other problems.

As the core index of the technology's advantages over traditional wired charging technology, the power transmission efficiency (PTE) of WPT system plays a decisive role in the large-scale application of the technology. Despite the WPT system works on the principle of high-frequency magnetic field propagating in the air, the large reluctance of the air (a relative permeability of only 1 ) greatly affects the PTE of the system. The main methods to improve the PTE of the system include: increasing the working frequency of the system, reducing the loss of the system itself, and increasing the coupling between the coils. As a rule, high operating frequency corresponds to the smaller limit index in the safety standard of electromagnetic environment [11], which may cause the electromagnetic environment around the system to fail to meet the limit index and thus fail to be normally applied. Also, a high frequency puts forward higher requirements for the design and stable operation of the inverter. In addition, 
restricted by the size, space and weight of the system, as well as the processing cost of magnetic materials, it is challenging to improve the coupling strength between coils, which justifies the feasibility of minimizing the resistance loss of the system itself. In general, the loss of the system comes from that of high-frequency inverter, the transceiver coil and the rectifier converter. In view of the large number of components involved in high-frequency inverters and rectifier conversion devices, apart from the optimal control of soft switches, it is expected to conduct optimal selection of devices in terms of hardware to reduce loss. Thus, the minimization of coil losses is believed to be feasible.

Since the Massachusetts Institute of Technology (MIT) proposed magnetic coupling resonance WPT technology in 2007 [1], academia and industry have done a lot of research for solving the problem of WPT system transmission distance and PTE improvement [12-16], and proposed frequency tracking [13], impedance matching [14], changing the coil structure, and adding magnetic circuit optimization structure $[15,16]$. These methods have improved the PTE of the system to a certain extent, but more focus on the principle of increasing the coupling between the coils. There is relatively little research on the calculation of the coil self-resistance in the field of WPT.

For the calculation of self- and mutual impedances in planar inductors, Hurley et al. [17] studied the variation of the mutual impedance of two coaxial circular filaments with frequency in the presence of a semi-infinite substrate. On this basis, the authors proposed a theoretical model for calculating the self-inductance and equivalent resistance of a circular filament on a substrate with a limited thickness [18], and studied the variation of the mutual impedance of two coaxial circular filaments with frequency.

Based on the above research, the research on the change law of the impedance of the heating coil on a substrate is more in-depth in the field of induction heating [19-23]. A set of analytical expressions was derived for the equivalent impedance in a planar circular induction heating system [19]. This method was extended to the case of multilayer substrates with finite thickness and infinite area [20]. A theory of inductance calculation of planar spiral windings was extended to determine the inductance of planar spiral windings shielded by a double-layer planar EM shield which consists of a layer of soft magnetic material and a layer of conductive material [21]. The variation trend of the equivalent impedance of the induction heating coil wound with Litz-wire on the substrate of finite thickness and infinite area was also analyzed [22,23].

However, previous studies have mostly focused on the variation rule of coil effective series resistance (ESR) with frequency when the substrate contains ferrite or aluminum plate, and few studies have focused on the influence of the substrate size changes on coil ESR. Although the change rule of coil ESR when there are multiple ferrite cores based on the method of simulation and theory is mentioned in [24], it is still not systematically studied with respect to the change rule of coil ESR when the thickness and area of substrate change. So, here, the influence of substrate size on the coil ESR, for single-layer and double-layer substrates, is studied. The purpose is to get the variation law of coil ESR at changing area and thickness and constant weight of the substrate and help the researchers for efficiently selecting the substrate and further improving the transmission efficiency of the system.

For the above purpose, the resistance of circular disk coils without substrate is firstly analyzed theoretically in this paper. Based on existing research, the theoretical analysis of the coil resistance of single-layer and double-layer substrate with finite thickness and infinite area is studied respectively, followed by a discussion on the shifts of calculation methods when the substrate thickness is close to the skin depth. Through the establishment of the actual model and the introduction of COMSOL Multiphysics finite element simulation and experimental measurement, this is compared with the theoretical calculation and summarized the variation rule of coil ESR at changing thickness of the substrate. Finally, the variation law of coil ESR at changing area and thickness and constant weight of the substrate is discussed to explore ways to reduce coil ESR.

The structure of this paper is as follows. In Section 2, the theoretical calculation method of the coil's conduction resistance and induction resistance with the single-layer and double-layer substrates is modeled and analyzed. In Section 3, the change rule of coil ESR when the size of the single-layer 
substrate changes is analyzed by theory, simulation, and experiment. When the size of the double-layer substrate changes, the change rule of coil ESR is also analyzed in Section 4. Section 5 concludes this paper.

\section{Modeling of Coil ESR}

The loss of the coil mainly includes the conduction resistance of the Litz-wire bundle itself and the induction resistance caused by the substrate material around the coil [25]. That is to say, coil ESR $R_{E S R}$ with substrate material consists of two parts: conduction resistance $R_{\mathrm{AC}}$ and substrate induction resistance $R_{\text {Ind }}$, which is defined as $R_{E S R}=R_{\mathrm{AC}}+R_{\text {Ind }}$. In order to reduce the coil loss as much as possible, it is not only necessary to realize the accurate calculation of the conducting resistance of the coil itself, but also to grasp the influence law of the substrate material on the change of the coil resistance. Firstly, the conduction resistance of the coil is modeled and analyzed in this section.

\subsection{Coil Conduction Resistance}

\subsubsection{Calculation of Litz-Wire Bundle Resistance}

In order to reduce the conduction resistance of the coil, multiple film-insulated round copper wires are often used to wind the coil by twisting into a Litz-wire bundle. The coil of WPT system works under the condition of high frequency current, and there is skin effect in the flow of high frequency current in film-insulated round copper wire. Therefore, after the working frequency of WPT system is determined, the diameter of single wire should be selected based on the skin depth of copper wire to ensure the optimization of cost and loss. Based on the recommendation of Litz-wire manufacturer, the model of the copper wire corresponding to the commonly used working frequency band is shown in Table 1. The specifications of the copper wire in Table 1 meet the NEMAMW1000 (Revision2008) standard.

Table 1. The relationship between the strand type and working frequency of Litz-wire.

\begin{tabular}{ccccc}
\hline $\begin{array}{c}\text { Working } \\
\text { Frequency Band }\end{array}$ & $\begin{array}{c}\text { AWG } \\
\text { Size }\end{array}$ & $\begin{array}{c}\text { Bare Wire Diameter } \\
\boldsymbol{d}_{\boldsymbol{s}}(\mathbf{m m})\end{array}$ & $\begin{array}{c}\text { Single Paint Film } \\
\text { Diameter } \boldsymbol{d}_{\boldsymbol{s}^{\prime}}(\mathbf{m m})\end{array}$ & $\begin{array}{c}\text { Unit Resistance } \\
\boldsymbol{R}_{\text {S_Unit }}(\boldsymbol{\Omega} / \mathbf{m})\end{array}$ \\
\hline $60-1000 \mathrm{~Hz}$ & 28 & 0.320 & 0.356 & 0.2123 \\
$1-10 \mathrm{kHz}$ & 30 & 0.254 & 0.284 & 0.3371 \\
$10-20 \mathrm{kHz}$ & 33 & 0.180 & 0.206 & 0.6689 \\
$20-50 \mathrm{kHz}$ & 36 & 0.127 & 0.147 & 1.350 \\
$50-100 \mathrm{kHz}$ & 38 & 0.102 & 0.119 & 2.111 \\
$100-200 \mathrm{kHz}$ & 40 & 0.079 & 0.094 & 3.519 \\
$200-350 \mathrm{kHz}$ & 42 & 0.064 & 0.076 & 5.421 \\
$350-850 \mathrm{kHz}$ & 44 & 0.051 & 0.061 & 8.495 \\
$850 \mathrm{k}-1.4 \mathrm{MHz}$ & 46 & 0.0399 & 0.0508 & 13.802 \\
$1.4-2.8 \mathrm{MHz}$ & 48 & 0.0315 & 0.0381 & 22.129 \\
\hline
\end{tabular}

For the resistance value of the Litz-wire bundle, the DC resistance $R_{\mathrm{DC}}$ is to be calculated firstly. Then the AC resistance $R_{\mathrm{AC}}$ is calculated in high frequency. According to [26], the accurate theoretical calculation of the DC resistance of the Litz-wire bundle is

$$
R_{\mathrm{DC}}=\frac{4 \rho l}{n_{\mathrm{Litz}} \pi d_{s}^{2}}\left(1+\frac{n_{\mathrm{Litz}} \pi^{2} d_{s}^{2}}{4 K_{a} P^{2}}\right)
$$


where $\rho$ is the resistivity of the Litz-wire strand, $l$ is the actual length of the Litz-wire strand, $P$ is the pitch of the Litz-wire bundle, $d_{S}$ is the bare wire diameter of the Litz-wire strand, $n_{\text {Litz }}$ is the strand number of the Litz-wire bundle, and $K_{a}$ is the packing factor of the Litz-wire bundle and

$$
\begin{gathered}
K_{a}=\frac{A_{c u}}{A_{b}}=\frac{n_{\mathrm{Litz}} d_{s}^{2}}{d_{\mathrm{b}}^{2}} \\
l=l_{\text {tot }} \sqrt{1+\left(\pi d_{b} / P\right)^{2}}
\end{gathered}
$$

where $A_{b}$ is the overall bundle area $\left(\pi d_{b}^{2} / 4\right), A_{c u}$ is the sum of the cross sectional areas of all the strands $\left(n_{\text {Litz }} \pi d_{s}^{2} / 4\right), d_{b}$ is the overall bundle diameter of the Litz-wire bundle and $d_{b} \approx d_{s^{\prime}} \times \sqrt{n_{\text {Litz }} / 0.75}, d_{s^{\prime}}$ is the paint film diameter of the Litz-wire strand, and $l_{\text {tot }}$ is the length of the Litz-wire bundle for coil.

Furthermore, according to [27], the accurate calculation equation of AC resistance of the Litz-wire bundle can be obtained by

$$
R_{\mathrm{AC}}=R_{\mathrm{DC}}\left(1+\frac{K_{a} n_{\mathrm{Litz}} \pi^{4} d_{s}^{4} f^{2} \times 10^{-14}}{8 \rho^{2}}\right)
$$

where $f$ is the frequency of current in Litz-wire bundle.

Of course, in the case of low error requirements, it might be estimated by the ratio of the unit resistance of a copper strand in the corresponding working frequency band in Table 1 to the number of strands of the Litz-wire bundle as

$$
R_{\mathrm{AC}} \approx R_{\mathrm{DC}}=l_{\text {tot }} \frac{R_{\mathrm{S} \_ \text {Unit }}}{n_{\text {Litz }}} .
$$

\subsubsection{Circumference Calculation of Disk Coil}

As can be seen from the Equation (3), the accurate calculation of the coil conduction resistance requires the total length of the Litz-wire bundle winding the coil, that is, the circumference of the coil. This paper calculates the length of the disk coil as shown in Figure 1. The number of turns is $N$, the minimum radius of the coil ring is $r_{\text {min }}$, and the maximum radius of the coil ring is $r_{\max }$ in the disk coil. The turn spacing of the coil is $\Delta D$.

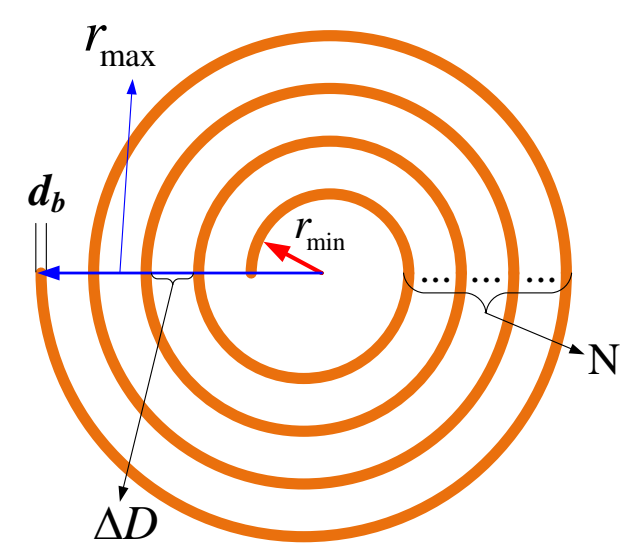

Figure 1. The Structure diagram of disk coil.

For a more accurate simulation of the actual winding of the coil, it is assumed that the disk coil is tangentially connected by a semi-arc with a gradually increasing diameter, and the diameter increases by $\Delta D+d_{b}$ with each additional semi-arc. Let the diameter of the first semi-arc of the first turn be $2 r_{\min }$, the diameter of the second semi-arc of the first turn is $2 r_{\min }+\Delta D+d_{b}$, the diameter of the first semi-arc of the second turn is $2 r_{\min }+\Delta D+d_{b}+\Delta D+d_{b}$ and the diameter of the second semi-arc 
of the second turn is $2 r_{\min }+\Delta D+d_{b}+\Delta D+d_{b}+\Delta D+d_{b}$. By analogy, the diameter of the second semi-arc of the $N$ th turn is $2 r_{\min }+(2 N-1)\left(\Delta D+d_{b}\right)$. In summary, the total length of the Litz-wire bundle winding the coil shall be

$$
l_{\text {tot }}=\frac{\pi}{2}\left[4 N r_{\min }+N(2 N-1)\left(\Delta D+d_{b}\right)\right]
$$

Therefore, the conduction resistance of the disk coil without substrate can be calculated by simultaneous Equations (1)-(4) and (6).

\subsection{Coil Induction Resistance with Substrate}

In order to meet the requirements of electromagnetic environment limits and improve the coupling between coils, substrate materials are often used around coils to increase coupling and shield electromagnetic fields. Due to the eddy current effect, the substrate material shields the magnetic field, but it also affects the resistance of the coil, i.e., induction resistance $R_{\text {Ind }}$. In this paper, the theoretical modeling calculation of induction resistance is carried out next.

\subsubsection{Coil Induction Resistance with Single-Layer Substrate}

The coil model with single-layer substrate is as shown in Figure 2. The diameter of the coil wire in it is the overall bundle diameter $d_{b}$ of the Litz-wire bundle. The outer radius of the outermost turn of the coil is $r_{10}$, and the inner radius is $r_{1 \mathrm{i}}$. The secondary outer turns are $r_{2 \mathrm{o}}$ and $r_{2 \mathrm{i}}$ respectively. The innermost turns are $r_{\mathrm{No}}$ and $r_{\mathrm{Ni}}$ respectively, and so on. $\mu_{r 1}$ and $\sigma_{1}$ are the relative permeability and conductivity of the substrate material, respectively. $t_{1}$ is the thickness of the substrate material. $d_{c}$ is the vertical distance from the center point of the cross section of the coil to the origin $\mathrm{O}$.

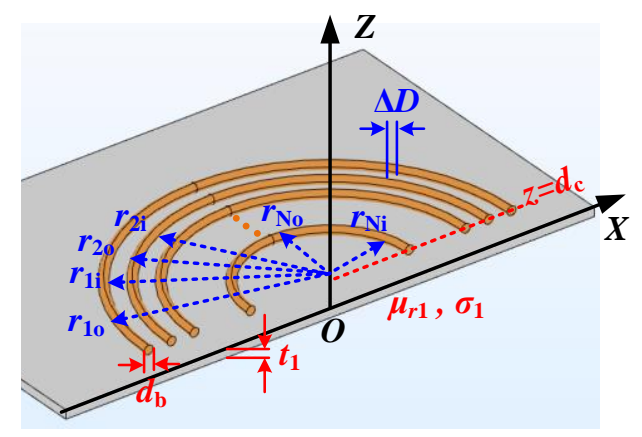

Figure 2. The coil model with single-layer substrate.

Based on the calculation equation of the mutual impedance of the coil with a finite thickness of substrate material in [18], the calculation equation of the coil induction resistance with single-layer substrate in Figure 2 can be obtained by

$$
R_{\text {Ind-1 }}=\operatorname{real}\left[\sum_{m=1}^{N} \sum_{n=1}^{N} \frac{j \omega \mu_{0} \pi}{d_{b}^{2} \ln \left(\frac{r_{\mathrm{mo}}}{r_{\mathrm{mi}}}\right) \ln \left(\frac{r_{\mathrm{no}}}{r_{\mathrm{ni}}}\right)} \int_{0}^{\infty} S\left(k r_{\mathrm{mo}}, k r_{\mathrm{mi}}\right) \cdot S\left(k r_{\mathrm{no}}, k r_{\mathrm{ni}}\right) Q\left(k d_{b}\right) \lambda\left(t_{1}\right) e^{-2 k d_{\mathrm{c}}} d k\right]
$$

where

$$
\begin{gathered}
S\left(k r_{\mathrm{mo}}, k r_{\mathrm{mi}}\right)=\frac{J_{0}\left(k r_{\mathrm{mo}}\right)-J_{0}\left(k r_{\mathrm{mi}}\right)}{k} \\
Q\left(k d_{b}\right)=\frac{2}{k}\left(d_{b}+\frac{e^{-k d_{b}}-1}{k}\right) \\
\lambda\left(t_{1}\right)=\phi(k) \frac{1-e^{-2 \eta t_{1}}}{1-\phi(k)^{2} e^{-2 \eta t_{1}}}
\end{gathered}
$$




$$
\begin{gathered}
\eta=\sqrt{k^{2}+j \omega \mu_{0} \mu_{r 1} \sigma_{1}} \\
\phi(k)=\frac{\mu_{\mathrm{r} 1} k-\eta}{\mu_{\mathrm{r} 1} k+\eta}
\end{gathered}
$$

$\mu_{0}$ is the permeability of free space $\left(4 \pi \times 10^{-7} \mathrm{H} / \mathrm{m}\right) . J_{0}(k r)$ is the first kind of 0 -order Bessel function.

The application condition of Equation (7) is that the thickness of substrate shall be less than 5 times of the skin depth of material. In case that the thickness of substrate fails to meet the condition, it might be equivalent to a semi-infinite plane and calculated by

$$
R_{\text {Ind-1 }}=\operatorname{real}\left[\sum_{m=1}^{N} \sum_{n=1}^{N} \frac{j \omega \mu_{0} \pi}{d_{b}^{2} \ln \left(\frac{r_{\mathrm{mo}}}{r_{\mathrm{mi}}}\right) \ln \left(\frac{r_{\mathrm{no}}}{r_{\mathrm{ni}}}\right)} \int_{0}^{\infty} S\left(k r_{\mathrm{mo}}, k r_{\mathrm{mi}}\right) \cdot S\left(k r_{\mathrm{no}}, k r_{\mathrm{ni}}\right) Q\left(k d_{b}\right) \phi(k) e^{-2 k d_{c}} d k\right]
$$

\subsubsection{Coil Induction Resistance with Double-Layer Substrate}

The electromagnetic shielding around the coil of WPT system usually adopts a double-layer substrate composed of ferrite and aluminum plate. That is, the first layer of substrate adjacent to the coil is ferrite, and the second layer is aluminum plate, as shown in Figure 3.

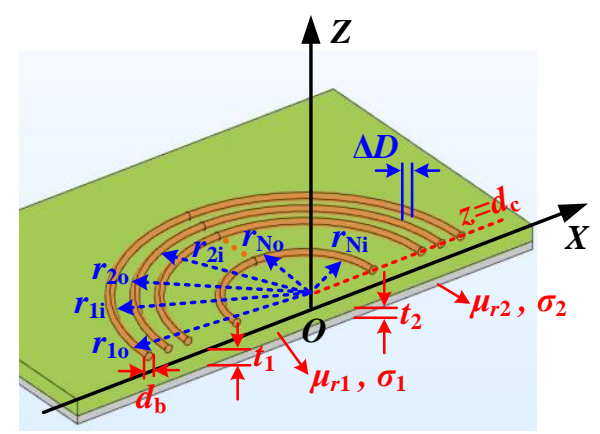

Figure 3. The coil model with double -layer substrate.

Compared with Figure 2, a layer of substrate material with thickness $t_{2}$, relative permeability $\mu_{r 2}$ and conductivity $\sigma_{2}$ is added. Based on the mutual impedance calculation equation of the coil with double-layer substrate material given in [21], the calculation equation of the coil induction resistance with a double-layer substrate material can be obtained as follows

$$
R_{\text {Ind-2 }}=\operatorname{real}\left[\sum_{m=1}^{N} \sum_{n=1}^{N} \frac{j \omega \mu_{0} \pi}{d_{b}^{2} \ln \left(\frac{r_{\mathrm{mo}}}{r_{\mathrm{mi}}}\right) \ln \left(\frac{r_{\mathrm{no}}}{r_{\mathrm{ni}}}\right)} \int_{0}^{\infty} S\left(k r_{\mathrm{mo}}, k r_{\mathrm{mi}}\right) \cdot S\left(k r_{\mathrm{no}}, k r_{\mathrm{ni}}\right) Q\left(k d_{b}\right) \lambda\left(t_{1}, t_{2}\right) e^{-2 k d_{c}} d k\right]
$$

where

$$
\begin{gathered}
\lambda\left(t_{1}, t_{2}\right)=\frac{\phi_{1}(k)+\frac{\theta\left(t_{2}\right)-m}{\theta\left(t_{2}\right)+m} e^{-2 \eta_{1} t_{1}}}{1+\phi_{1}(k) \frac{\theta\left(t_{2}\right)-m}{\theta\left(t_{2}\right)+m} e^{-2 \eta_{1} t_{1}}} \\
\theta\left(t_{2}\right)=\frac{1-\phi_{2}(k) e^{-2 \eta_{2} t_{2}}}{1+\phi_{2}(k) e^{-2 \eta_{2} t_{2}}} \\
m=\frac{\mu_{r 1} \eta_{2}}{\mu_{r 2} \eta_{1}} \\
\eta_{1}=\sqrt{k^{2}+j \omega \mu_{0} \mu_{r 1} \sigma_{1}} \\
\eta_{2}=\sqrt{k^{2}+j \omega \mu_{0} \mu_{r_{2}} \sigma_{2}}
\end{gathered}
$$




$$
\begin{aligned}
& \phi_{1}(k)=\frac{\mu_{\mathrm{r} 1} k-\eta_{1}}{\mu_{\mathrm{r} 1} k+\eta_{1}} \\
& \phi_{2}(k)=\frac{\mu_{\mathrm{r} 2} k-\eta_{2}}{\mu_{\mathrm{r} 2} k+\eta_{2}}
\end{aligned}
$$

All of the above parameters are in SI units. To sum up, this paper has modeled the calculation method of the equivalent resistance of the coil with single-layer and double-layer substrate materials. Next, the above method is verified by some examples, and the influence of substrate material size change on coil ESR is discussed.

\section{Analysis of Coil ESR with Single-Layer Substrate}

The coil as shown in Figure 4 is used for analysis in this paper, and its detailed parameters are shown in Table 2. The Litz-wire of the winding coil is insulated with its own paint film layer and external woolen material. The substrate around the coil to shield the electromagnetic field is usually made of ferrite, aluminum and other materials [15], whose conductivity and permeability are shown in Table 3. Among them, the conductivity of Ferrite 1 is $0 \mathrm{~S} / \mathrm{m}$, which is the ideal ferrite. The conductivity of Ferrite 2 is $10 \mathrm{~S} / \mathrm{m}$, which is closer to reality. Due to the common charging frequency of EVs wireless charging system is $85 \mathrm{kHz}$. Therefore, this paper focuses on the study of the influence of substrate material on coil ESR at $85 \mathrm{kHz}$. The skin depth of substrate materials at $85 \mathrm{kHz}$ is also listed in Table 3 .

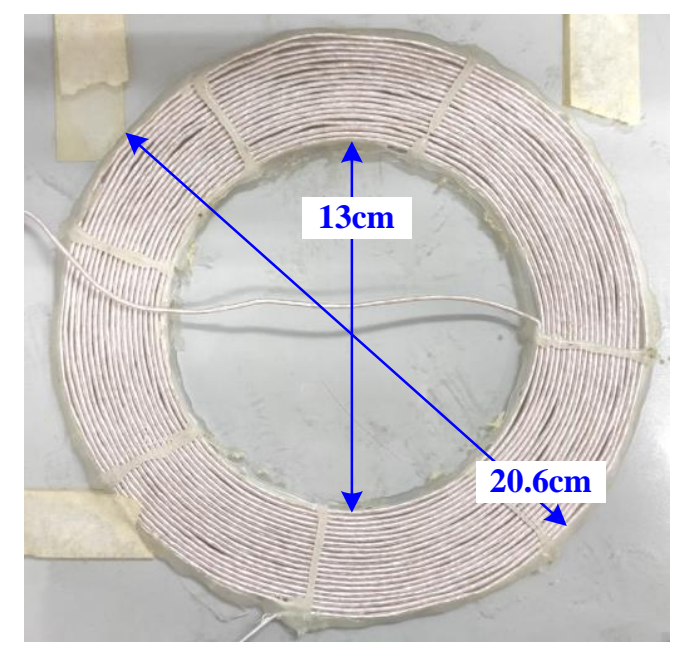

Figure 4. The prototype of coil.

Table 2. The mechanical parameters of the coil.

\begin{tabular}{cccc}
\hline Parameters & Value & Parameters & Value \\
\hline the strand diameter $d_{s}$ & $0.1 \mathrm{~mm}$ & strands number $n_{\text {Litz }}$ & 120 \\
the overall bundle diameter $d_{b}$ & $1.5 \mathrm{~mm}$ & coil turn spacing $\Delta D$ & $0.5 \mathrm{~mm}$ \\
coil inner diameter $2 r_{\text {min }}$ & $13 \mathrm{~cm}$ & coil outer diameter $2 r_{\text {max }}$ & $20.6 \mathrm{~cm}$ \\
coil turns $N$ & 19 & current flow area & $1 \mathrm{~mm}^{2}$ \\
\hline
\end{tabular}

Table 3. The substrate material parameters.

\begin{tabular}{cccc}
\hline Parameters & Ferrite 1 & Ferrite 2 & Aluminum \\
\hline Relative permeability $\mu_{\mathrm{r}}$ & 3300 & $3300 \pm 25 \%$ & 1 \\
Conductivity $\sigma(\mathrm{S} / \mathrm{m})$ & 0 & 10 & $3.82 \times 10^{7}$ \\
Skin depth @ $85 \mathrm{kHz}(\mathrm{mm})$ & $/$ & 545.9 & 0.2793 \\
\hline
\end{tabular}


Then, the change law of coil ESR is compared and analyzed by the methods of theory, finite element simulation software (Figure 5a) and experimental test (Figure 5b). The condition when the side length of substrate is far larger than the diameter of the outer ring of the coil is first analyzed.

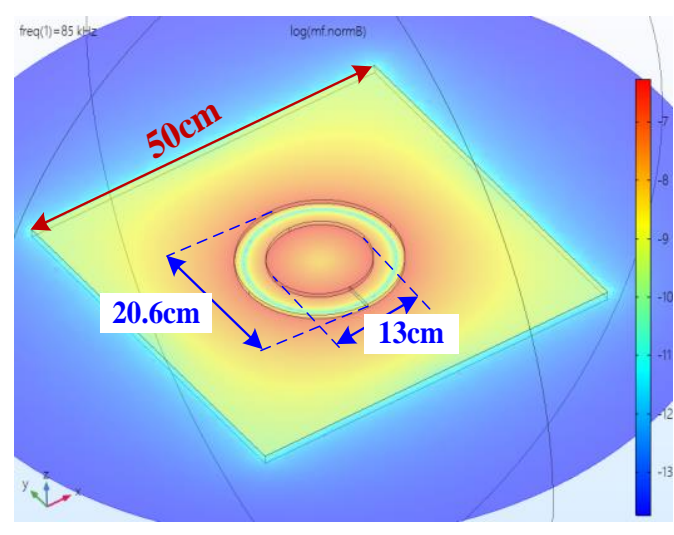

(a)

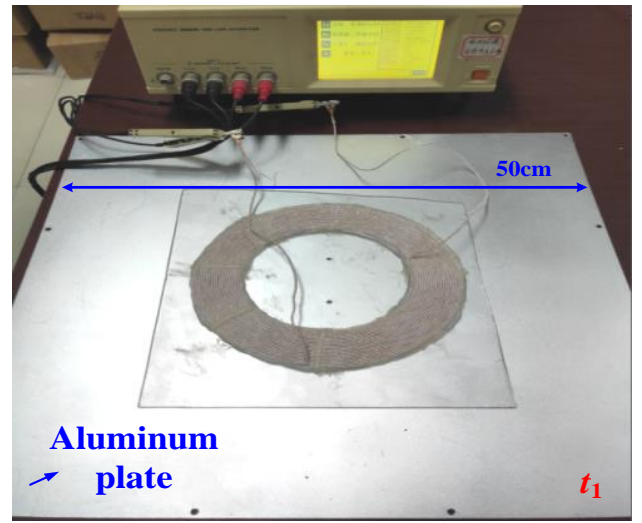

(b)

Figure 5. The model of coil with single-layer substrate: (a) Simulation model in COMSOL Multiphysics; (b) Experimental test platform.

\subsection{When the Thickness of Substrate Material Changes}

The theoretical calculation curve of coil ESR variation shall be obtained by substituting the data in Table 3 into Equations (4) and (7), i.e., the dot curve in Figure 6. Please note that a thickness of $1 \mathrm{~mm}$ of the aluminum plate, it should be calculated by Equation (7) because it is less than five times of skin depth. For the thickness of 2-10 mm, Equation (13) shall be adopted because it is greater than five times of skin depth.

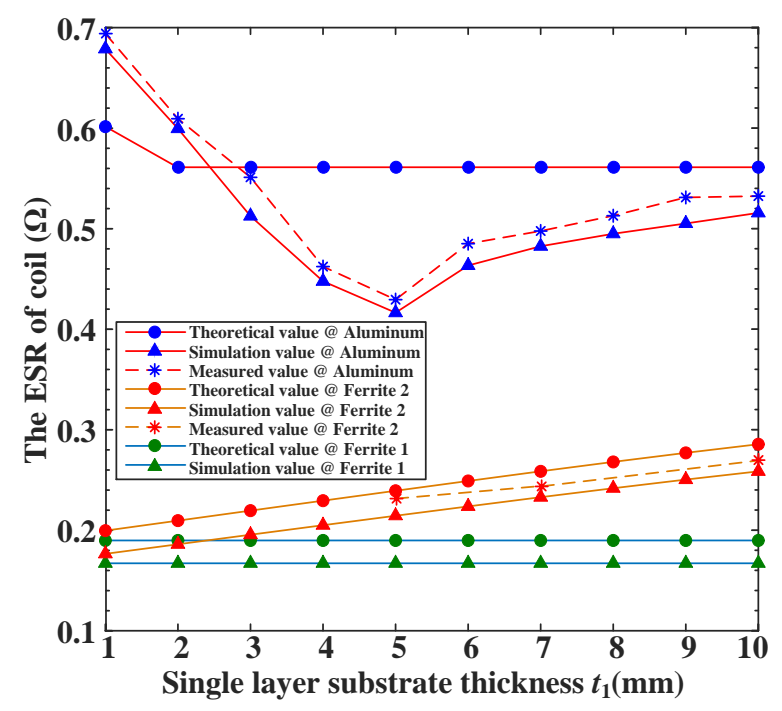

Figure 6. Coil effective series resistance (ESR) change curves when the thickness of single-layer substrate material changes.

The simulation model as shown in Figure 5a is established by setting the current flow area of a single conductor of $1 \mathrm{~mm}^{2}$ in COMSOL Multiphysics Coil Domain node and the substrate area of $50 \mathrm{~cm} \times 50 \mathrm{~cm}$ (i.e., the side length of $50 \mathrm{~cm}$, which meets the requirement of far larger than the diameter of the outer ring of the coil). The finite element calculation curve of coil ESR, i.e., the trigonometric curve in Figure 6, is obtained by parameterized scanning of the thickness of substrate. HIOKI 3532-50 LCR HiTESTER (HIOKI, Shanghai, China) (LCR analyzer) is introduced to build the experimental 
measurement platform in Figure 5b, and the measurement results of the variation of coil ESR including single-layer substrate are shown in the dotted line in Figure 6. Although Ferrite 1 with conductivity of 0 in Table 3 is an ideal material, it does not exist in reality, the experimental measurement curve of which is therefore not included in Figure 6. In addition, due to the complex processing technology of ferrite, only the ferrite with thickness of $5 \mathrm{~mm}, 7 \mathrm{~mm}$, and $10 \mathrm{~mm}$ is available, which is thus measured in this paper.

As the curve in Figure 6 implies, the theoretical calculation, finite element simulation calculation and experimental measurement results of ferrite are highly consistent. The change of thickness of Ferrite 1 has no effect on coil ESR. Coil ESR increases with the increase of the thickness of Ferrite 2, and the resistance increases by $31.73 \%$ at the thickness of $10 \mathrm{~mm}$ compared with at the thickness of $1 \mathrm{~mm}$, suggesting that the conductivity of substrate contributes to the main growth of coil ESR.

Also, according to Figure 6, coil ESR first decreases and then increases with the increase of the aluminum plate thickness. Coil ESR decreases to the lowest at the thickness of $5 \mathrm{~mm}$ of the aluminum plate substrate, which is a decrease of $38.66 \%$ compared with that at the thickness of $1 \mathrm{~mm}$ and a decrease of $19.25 \%$ compared with that at the thickness of $10 \mathrm{~mm}$. The variation might be attributed to the combination of eddy current and skin effect in aluminum plate. In spite of the high consistency of the results of finite element simulation and experimental measurement of coil ESR of single-layer aluminum plate, there are significant deviations between the theoretical calculation results and the simulation and experimental results. This may be due to the assumptions of the theoretical calculation model are in the transition stage when the thickness of the aluminum plate is 5-50 times of the skin depth (i.e., $1-7 \mathrm{~mm}$ ). Therefore, in the coil design and optimization analysis, a simple assessment of resistance loss might be made by theoretical calculation before a more accurate evaluation of resistance by the Coil Domain calculation of COMSOL Multiphysics, thus improving the complex calculation in the evaluation of equivalent resistance in coil design.

From the above, when the size of single-layer substrate is far larger than that of coil, shielding the magnetic field with materials with the smallest conductivity is one of the effective ways to reduce coil ESR. Due to the extremely high conductivity of substrate materials (such as aluminum), the increase of thickness can be resorted to in case of high requirement for the shielding effect, whereas there is an optimal value of thickness. The thickness of substrate shall be appropriately selected based on a comprehensive consideration of coil weight and working performance.

According to the analysis above, for aluminum plate with finite thickness and infinite area, the theoretical calculation results are low consistency with experimental measurement. The experimental measurement results are high consistency with COMSOL Multiphysics simulation calculation. Therefore, the methods of simulation and experimental measurement shall be adopted to explore the variation rule of coil ESR at changing area and volume of substrate.

\subsection{When the Area of Substrate Material Changes}

Based on the three materials in Table 3, the change of coil ESR when the area of different substrate materials changes from $5 \mathrm{~cm} \times 5 \mathrm{~cm}$ to $26 \mathrm{~cm} \times 26 \mathrm{~cm}$, i.e., the side length changes from $5 \mathrm{~cm}$ to $26 \mathrm{~cm}$, is calculated by simulation, as shown in Figure 7. In Figure 7a, the red curve is the change curve of coil ESR when the substrate material is Ferrite 1, and the blue curve is the change curve of coil ESR when the substrate material is Ferrite 2. Figure $7 \mathrm{~b}$ is the change curve of coil ESR when the substrate material is aluminum plate. 


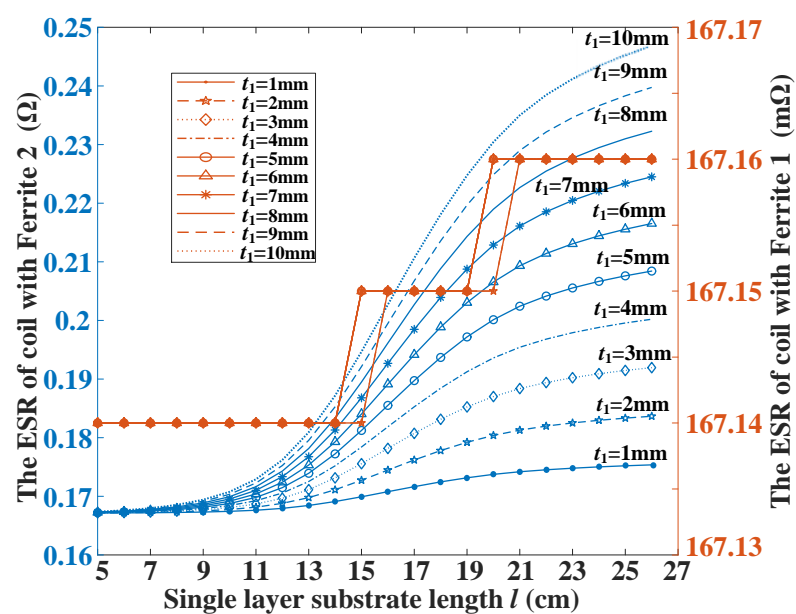

(a)

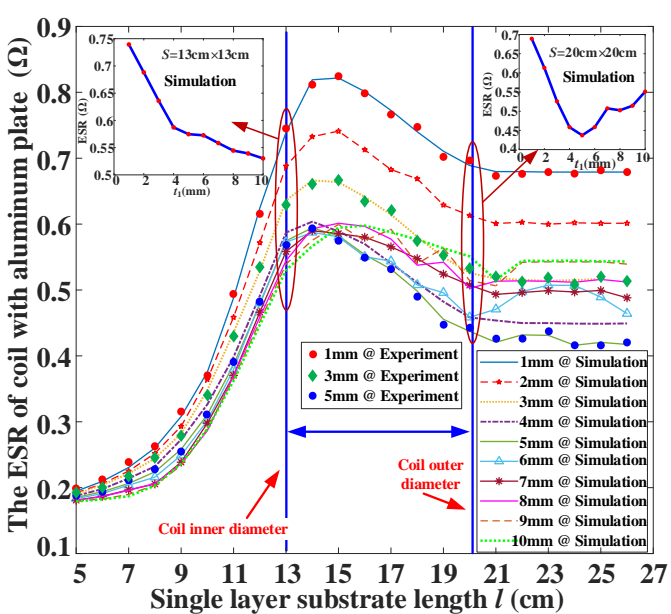

(b)

Figure 7. Coil ESR change curves when the area of single-layer substrate material changes: (a) With Ferrite 1 and Ferrite 2; (b) With aluminum plate.

As shown in Figure 7a, at changes of the thickness and area of Ferrite 1, coil ESR changes only within $0.01 \mathrm{~m} \Omega$, that is negligible, suggesting that the change of Ferrite 1 in area and thickness shall not affect coil ESR. At the constant thickness of Ferrite 2, coil ESR increases with the increase of area. At the thickness of $1 \mathrm{~mm}$, coil ESR of that with the area of $26 \mathrm{~cm} \times 26 \mathrm{~cm}$ increases by $4.92 \%$ compared that with the area of $5 \mathrm{~cm} \times 5 \mathrm{~cm}$. At the thickness of $10 \mathrm{~mm}$, coil ESR of that with the area of $26 \mathrm{~cm} \times 26 \mathrm{~cm}$ increases by $47.49 \%$ compared that with the area of $5 \mathrm{~cm} \times 5 \mathrm{~cm}$. At the constant area of Ferrite 2, the change of coil ESR is positively correlated with the increase of the thickness of Ferrite 2 . At the area of $11 \mathrm{~cm} \times 11 \mathrm{~cm}$, coil ESR of that with the thickness of $10 \mathrm{~mm}$ increases by $3.15 \%$ compared that with the thickness of $1 \mathrm{~mm}$. At the area of $26 \mathrm{~cm} \times 26 \mathrm{~cm}$, coil ESR of that with the thickness of $10 \mathrm{~mm}$ increases by $40.77 \%$ compared that with the thickness of $1 \mathrm{~mm}$. Therefore, in terms of practical application, on the premise of achieving mutual inductance between coils, coil ESR should be reduced by minimizing the thickness and area of Ferrite 2 .

From the curve in Figure $7 \mathrm{~b}$, it can be concluded that the effect of aluminum plate substrate on coil ESR is different from that of ferrite. Coil ESR decreases with the increase of the thickness of the aluminum plate when the side length of the aluminum plate is less than $13 \mathrm{~cm}$ (the diameter of the inner ring of the coil), and increases with the increase of the area of the aluminum plate at the same thickness. At the area of $9 \mathrm{~cm} \times 9 \mathrm{~cm}$, Coil ESR of that at the thickness of $10 \mathrm{~mm}$ is $21.99 \%$ lower than that of $1 \mathrm{~mm}$ thickness. At the thickness of $5 \mathrm{~mm}$, coil ESR of that at the area of $13 \mathrm{~cm} \times 13 \mathrm{~cm}$ is $210.6 \%$ higher than that of $5 \mathrm{~cm} \times 5 \mathrm{~cm}$.

As the side length of the aluminum plate continues to increase from $13 \mathrm{~cm}$, coil ESR shall gradually increase to the highest before starting decrease and finally tend to be stable. At the thickness of 1-2 mm, coil ESR is much greater than that of other aluminum plates with the same area. At the area of $20 \mathrm{~cm} \times 20 \mathrm{~cm}$, coil ESR of $1 \mathrm{~mm}$ thickness is $57.57 \%$ larger than that of $5 \mathrm{~mm}$ thickness and $25.02 \%$ larger than that of $10 \mathrm{~mm}$ thickness. Coil ESR of the aluminum plate with the thickness of $5 \mathrm{~mm}$ is smaller than that of the other plates with the same area when the side length of the aluminum plate with the diameter of $20 \mathrm{~cm}$ is close to the outer ring of the coil. Thereafter, with the increase of aluminum plate area, there is basically no change to coil ESR, and it remains the smallest at the thickness of $5 \mathrm{~mm}$.

According to the small diagram in the upper right corner of Figure $7 \mathrm{~b}$, at the area of $20 \mathrm{~cm} \times 20 \mathrm{~cm}$ of the aluminum plate, coil ESR first decreases and then increases with the increase of the aluminum plate thickness, and reaches the minimum at the thickness of $5 \mathrm{~mm}$. This implies that, when the side length of the aluminum plate is not less than the diameter of the outer ring of the coil, the value of coil ESR is the smallest at the thickness of $5 \mathrm{~mm}$ of the aluminum plate, which however is unavailable in 
reality due to the limitations of the application environment. This is suggested to take into account the appropriate thickness and area of aluminum plate substrate based on the system performance, coil size and shielding requirements.

Since the weight of the whole packaged coil is one of the major concerns in the industrial application of WPT system, the variation rule of coil ESR in case of constant weight and changing thickness and area of single-layer substrates shall be studied.

\subsection{When the Weight of Substrate Material is Constant}

At the constant substrate density, the constancy of weight might be equivalent to the constancy of volume. According to Section 3.1, in the area of $20 \mathrm{~cm} \times 20 \mathrm{~cm}$, the volume of $5 \mathrm{~mm}, 7 \mathrm{~mm}$, and $10 \mathrm{~mm}$ thickness are taken as the reference respectively, that is, to maintain a constant volume of $V=200 \mathrm{~cm}^{3}$, $V=280 \mathrm{~cm}^{3}$, and $V=400 \mathrm{~cm}^{3}$. The simulation software is adopted to calculate the variation of coil ESR at the thickness of 1-10 mm of the three materials in Table 3, as shown in Figure 8.

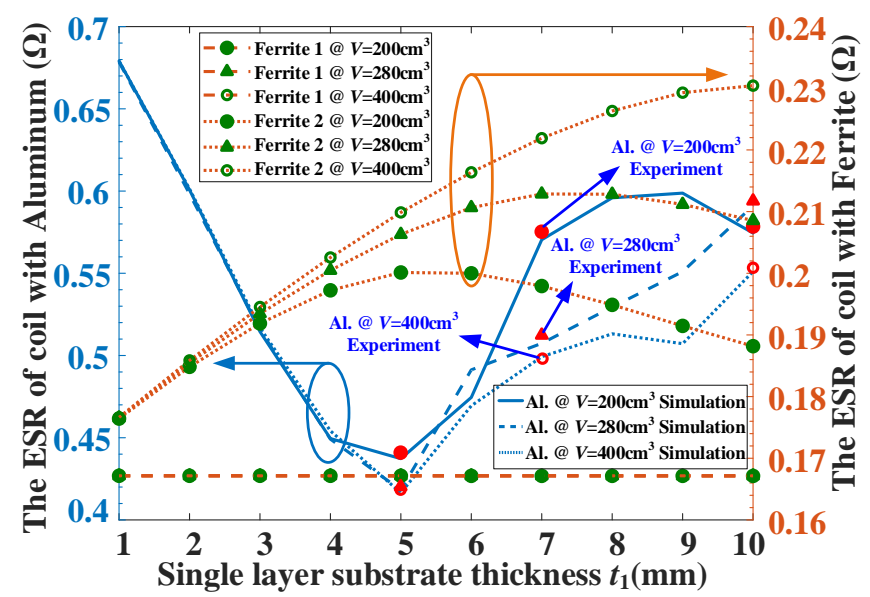

Figure 8. Coil ESR change curves when the weight of single-layer substrate material is constant.

As shown in Figure 8, Ferrite 1 with the same volume and different thickness has basically no effect on coil ESR. In the case of constant volume of Ferrite 2, coil ESR increases first and then decreases with the increase of thickness, and there is a maximum value. The maximum value of coil ESR of each volume appears at the area of $20 \mathrm{~cm} \times 20 \mathrm{~cm}$, i.e., at the thickness of $5 \mathrm{~mm}, 7 \mathrm{~mm}$, and $10 \mathrm{~mm}$, respectively. However, for a single-layer aluminum plate substrate, with the increase of thickness, coil ESR decreases first and then increases at the constant volume. Coil ESR of the three reference volumes is the smallest at the thickness $5 \mathrm{~mm}$ and the area of $20 \mathrm{~cm} \times 20 \mathrm{~cm}$ of the aluminum plate.

Therefore, at the constant volume, the closer the side length of Ferrite 2 is to the diameter of the coil outer ring, the larger coil ESR is and the larger the variation is. The minimum value of coil ESR of aluminum plate substrate appears at the thickness of $5 \mathrm{~mm}$ aluminum plate. To sum up, when the coil transmission performance and weight requirements shall be met, the smaller the thickness and the larger the area of Ferrite 2 are, and the closer the thickness of aluminum plate is to $5 \mathrm{~mm}$, the better for single-layer substrates.

\section{Analysis of Coil ESR with Double-Layer Substrate}

In this section, the effect of double-layer substrate composed of different materials on coil ESR shall be identified. There are two types of combinations of double-layer substrate, namely Aluminum plate + Ferrite 1 and Aluminum plate + Ferrite 2. Based on the coil shown in Figure 4, the simulation and experiment platform, as shown in Figure 9, is established to calculate coil ESR and the results are compared with the theoretical calculation results. 


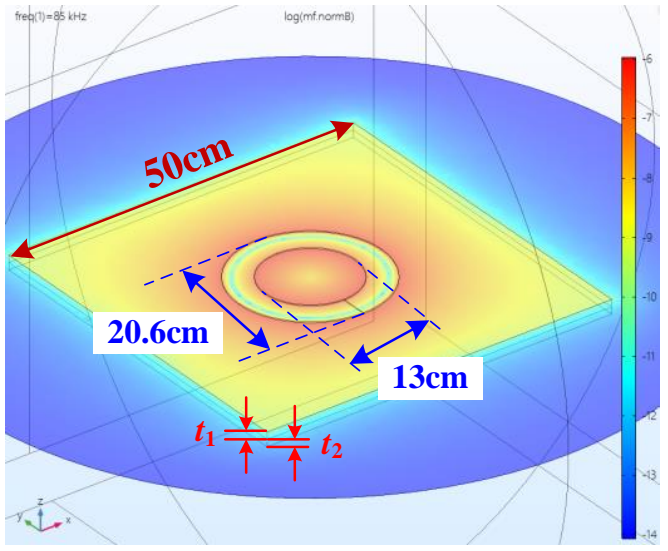

(a)

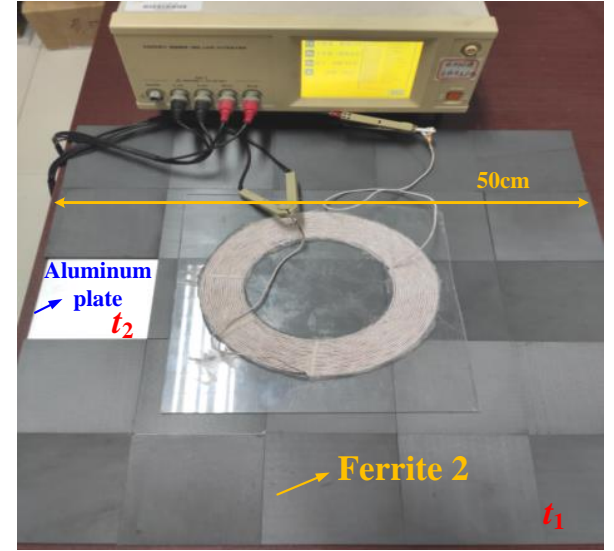

(b)

Figure 9. The model of coil with double-layer substrate: (a) Simulation model in COMSOL; (b) Experimental test platform.

\subsection{When the Thickness of Double-Layer Substrate Material Changes}

Similar to Section 3.1, we first analyze the case when the side length of the double-layer substrate is far larger than the diameter of the outer ring of the coil. On account of the data in Tables 2 and 3, when the thickness of each layer in the double-layer substrate varies between $1 \mathrm{~mm}$ and $10 \mathrm{~mm}$, Equation (14) is adopted to carry out the theoretical calculation of coil ESR to obtain the theoretical value curve of coil ESR, i.e., the orange curve in Figure 10. COMSOL Multiphysics is introduced to build the simulation model in Figure 9a, which is used to conduct parametric scanning of the substrate's thickness and obtain the simulation value curve of the variation of coil ESR. At the same time, LCR analyzer is introduced to build the experimental platform in Figure 9b. Similar to the ferrite in single-layer substrates, only the variation curve of coil ESR in the first layer of Ferrite 2 in double-layer substrate is measured at the thickness of $5 \mathrm{~mm}, 7 \mathrm{~mm}$, and $10 \mathrm{~mm}$. To conduct a more intuitive comparison with the simulation value and the measured value, only the case of "Aluminum plate + Ferrite 2" is shown in Figure 10.

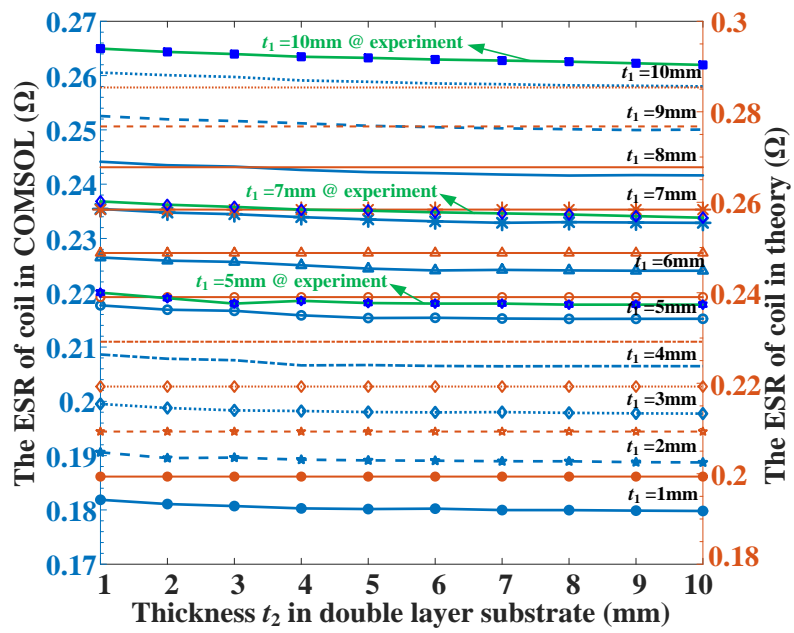

Figure 10. Coil ESR change curves when the thickness of double-layer substrate material changes.

As indicated by the abscissa in Figure 10, at the constant thickness of ferrite in the first layer of double-layer substrate, the change in the thickness of aluminum plate in the second layer of substrate has little effect on coil ESR. That is to say, when the double-layer substrate is large enough and the thickness of ferrite substrate in the first layer is determined, the change in the thickness of aluminum 
plate substrate in the second layer has no effect on coil ESR. According to the ordinate in Figure 10, coil ESR increases with the increase of the thickness of Ferrite 2 when the thickness of aluminum plate in the second layer of double-layer substrate is constant. At the thickness of $t_{2}=5 \mathrm{~mm}$ of aluminum plate in the second layer of double-layer substrate, coil ESR at $t_{1}=10 \mathrm{~mm}$ thickness of substrate in the first layer is $43.68 \%$ larger than that at $t_{1}=1 \mathrm{~mm}$ thickness. Therefore, it is suggested to minimize the thickness of ferrite substrate in the first layer on the premise of achieving the mutual inductance and shielding requirements between coils.

It can also be seen from Figure 10 that the coil ESR is almost unaffected by the change of thickness $t_{2}$. So, the coil ESR change law of theoretical, simulation and experimental results are a high consistency, when the size of the double-layer substrate is infinite compared with the coil. Nevertheless, the theoretical calculation is only applicable to the cases where the side length of the substrate is far larger than the diameter of the outer ring of the coil. When the side length of the substrate is close to the diameter of the outer ring of the coil, the next step of this paper is to analyze and study by combining simulation and measurement.

\subsection{When the Area of Double-Layer Substrate Material Changes}

In the case that the substrate size is close to that of the coil, the impact of the change in the area of double-layer substrate on coil ESR at the ferrite thickness of $5 \mathrm{~mm}, 7 \mathrm{~mm}$, and $10 \mathrm{~mm}$ shall be studied in this section based on the actual situation. The six types of combinations of double-layer substrate to be studied in this section are as shown in Table 4.

Table 4. The combinations parameters of double layer substrate.

\begin{tabular}{ccccc}
\hline Combinations & $\begin{array}{c}\text { 1st Layer } \\
\text { Material }\end{array}$ & $\begin{array}{c}\text { 1st Layer } \\
\text { Thickness }\end{array}$ & $\begin{array}{c}\text { 2nd Layer } \\
\text { Material }\end{array}$ & 2nd Layer Thickness \\
\hline 1 & Ferrite 2 & $5 \mathrm{~mm}$ & Aluminum & $1 \mathrm{~mm} \leq t_{2} \leq 10 \mathrm{~mm}$ \\
2 & Ferrite 1 & $5 \mathrm{~mm}$ & Aluminum & $1 \mathrm{~mm} \leq t_{2} \leq 10 \mathrm{~mm}$ \\
3 & Ferrite 2 & $7 \mathrm{~mm}$ & Aluminum & $1 \mathrm{~mm} \leq t_{2} \leq 10 \mathrm{~mm}$ \\
4 & Ferrite 1 & $7 \mathrm{~mm}$ & Aluminum & $1 \mathrm{~mm} \leq t_{2} \leq 10 \mathrm{~mm}$ \\
5 & Ferrite 2 & $10 \mathrm{~mm}$ & Aluminum & $1 \mathrm{~mm} \leq t_{2} \leq 10 \mathrm{~mm}$ \\
6 & Ferrite 1 & $10 \mathrm{~mm}$ & Aluminum & $1 \mathrm{~mm} \leq t_{2} \leq 10 \mathrm{~mm}$ \\
\hline
\end{tabular}

The results in Figure 11 are obtained by introducing finite element simulation and experimental measurements. The abscissa represents the change of area with the change of side length of double-layer substrate in Figure 11.

It can be concluded from Figure 11 that, when the side length of the substrate is smaller than the diameter of the inner ring of the coil, coil ESR gradually reaches a peak with the increase of the side length of the substrate. Coil ESR decreases and tends to be stable when the side length of substrate continues to increase to be larger than the diameter of outer coil ring. In Figure 11a,c,e, when the thickness of the aluminum plate is $5 \mathrm{~mm}$ and the first layer thickness of double-layer substrate is $5 \mathrm{~mm}$, $7 \mathrm{~mm}$, and $10 \mathrm{~mm}$ respectively, coil ESR of that with the area of $26 \mathrm{~cm} \times 26 \mathrm{~cm}$ decreases by $53.34 \%$, $39.52 \%$, and $31.46 \%$ compared that with the area of $14 \mathrm{~cm} \times 14 \mathrm{~cm}$. That is to say, with the constant thickness of double-layer substrate, coil ESR increases first and then decrease with the increase of the substrate area. Therefore, when the thickness of double-layer substrate is constant and the side length is larger than the diameter of the inner ring of the coil, the continuous increase of the side length of double-layer substrate shall effectively reduce coil ESR.

At the constant side length of double-layer substrate, coil ESR decreases with the increase of the thickness at the thickness of $1-5 \mathrm{~mm}$ of the aluminum plate. When the thickness of aluminum plate is more than $5 \mathrm{~mm}$, coil ESR hardly changes with the thickness. Therefore, increase of the thickness of aluminum plate in double-layer substrate shall reduce coil ESR when the side length of the substrate is constant. 


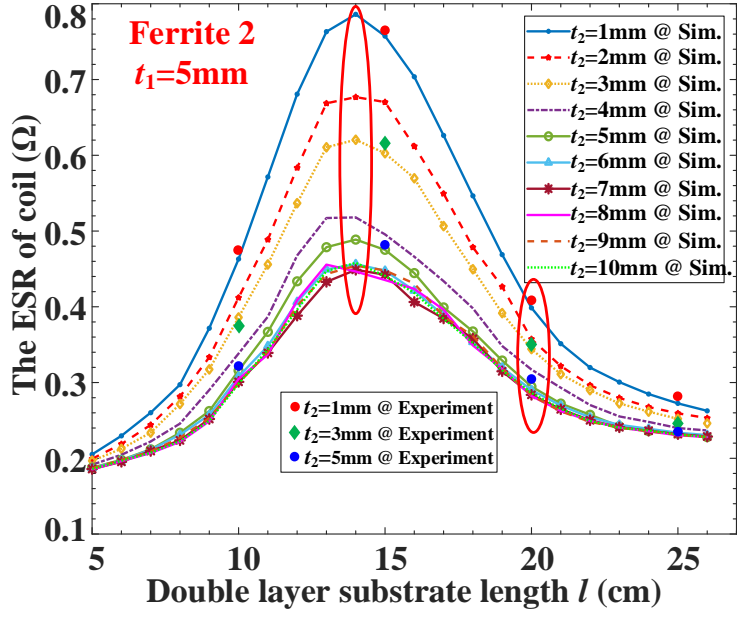

(a)

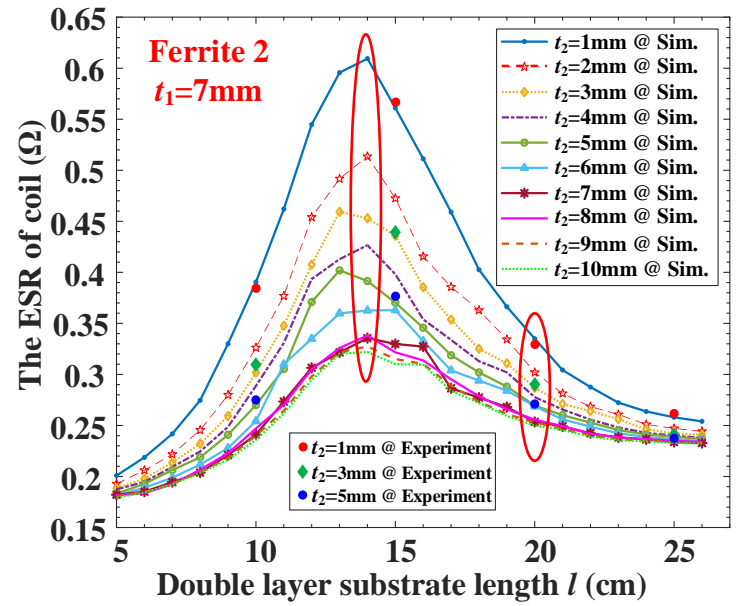

(c)

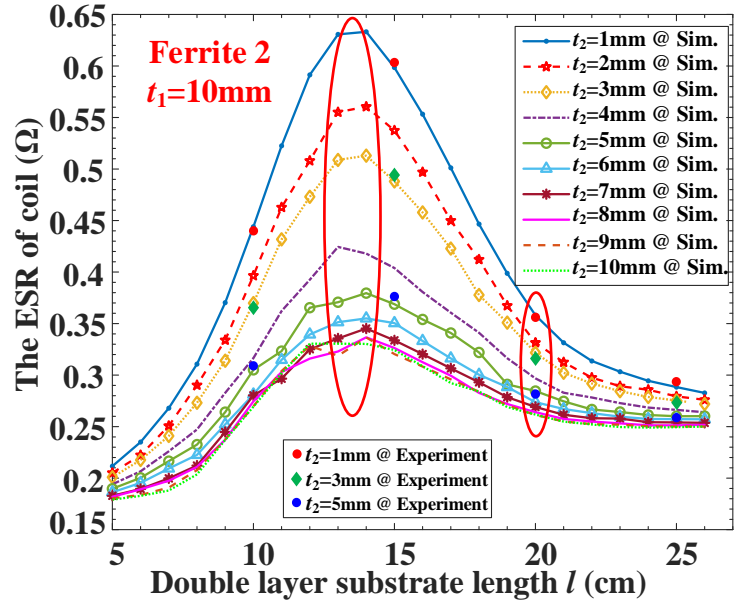

(e)

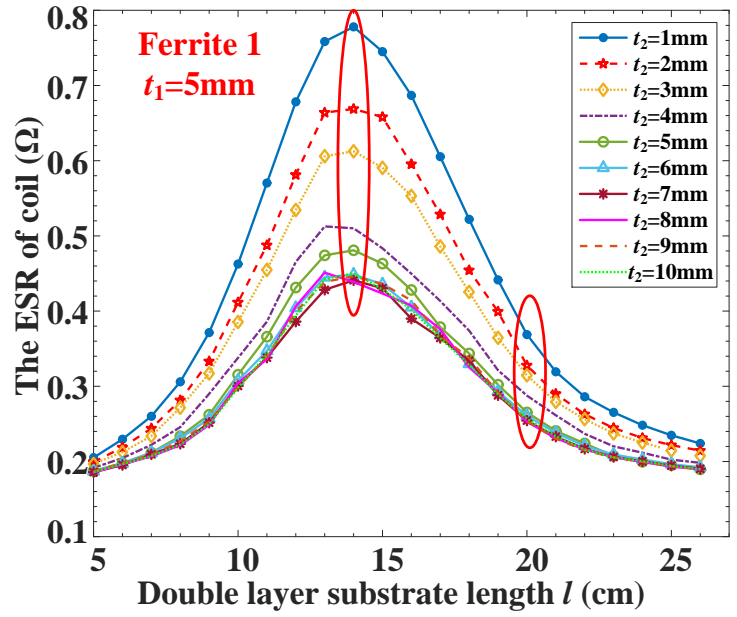

(b)

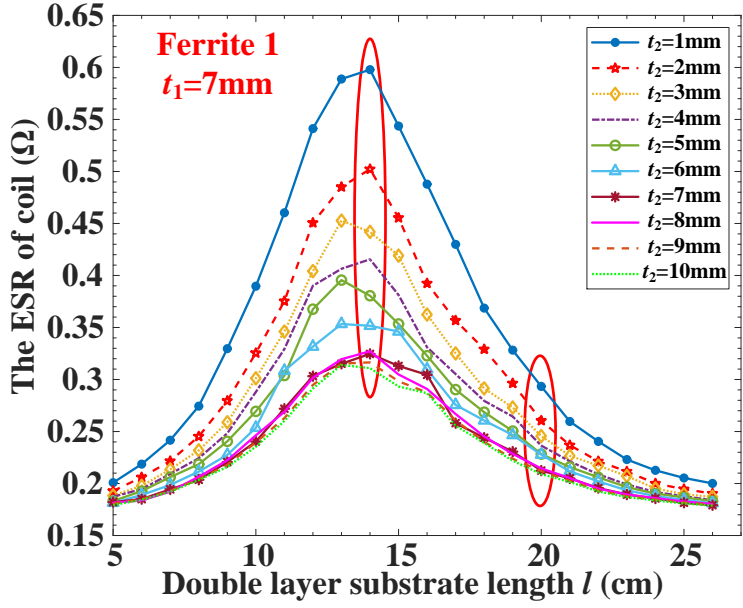

(d)

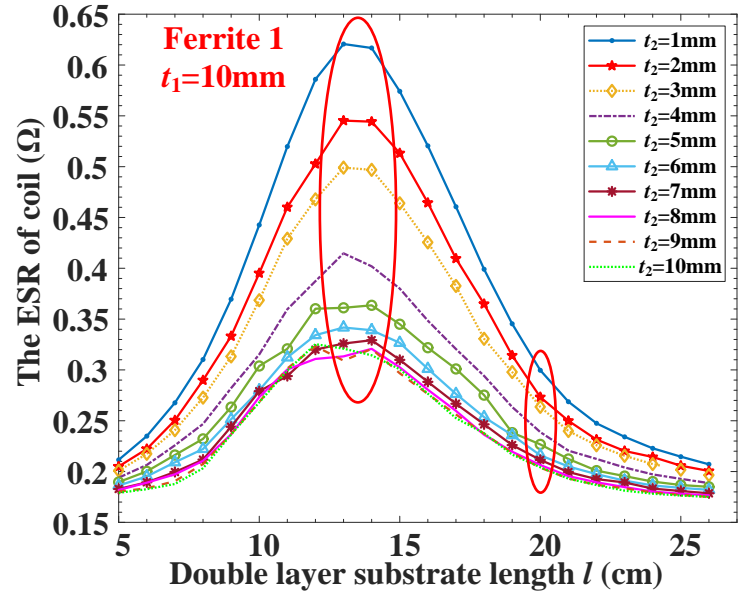

(f)

Figure 11. Coil ESR change curves when the area of double-layer substrate material changes: (a) Combination 1; (b) Combination 2; (c) Combination 3; (d) Combination 4; (e) Combination 5; (f) Combination 6 .

Compared with Ferrite 1, with the same thickness of ferrite in the first layer of double-layer substrate, there is almost no change to coil ESR, suggesting that coil ESR is mainly affected by the aluminum plate in double-layer substrate. When the side length of double-layer substrate is larger than the diameter of the coil outer ring and the thickness of the aluminum plate is constant, the double-layer 
substrate with Ferrite 1 assumes smaller impact on coil ESR. When the thickness of the aluminum plate is $5 \mathrm{~mm}$, the first layer of double-layer substrate is respectively $5 \mathrm{~mm}, 7 \mathrm{~mm}$ and $10 \mathrm{~mm}$ thick and has an area of $26 \mathrm{~cm} \times 26 \mathrm{~cm}$, coil ESR of Ferrite 1 is $16.86 \%, 22.68 \%$, and $28.91 \%$ smaller than that of Ferrite 2 . That is, ferrite with lower conductivity might be employed to reduce coil ESR when the size of double-layer substrate is larger than the diameter of the outer ring of coil.

In summary, for a double-layer substrate, coil ESR shall be reduced by increasing the side length of the substrate, increasing the thickness of aluminum plate substrate in the second layer and reducing the conductivity of ferrite in the first layer. Similar to the analysis of a single-layer substrate, research on a double-layer substrate at constant weight shall be carried out as follows.

\subsection{When the Weight of Double-Layer Substrate Material is Constant}

For double-layer substrate materials, the constant weight can also be equivalent to the constant material volume. In the area of $20 \mathrm{~cm} \times 20 \mathrm{~cm}$, the volume of $5 \mathrm{~mm}$ and $7 \mathrm{~mm}$ thickness are also taken as the reference respectively, that is, to maintain a constant volume of $V=200 \mathrm{~cm}^{3}, V=280 \mathrm{~cm}^{3}$ and $V=400 \mathrm{~cm}^{3}$. The variation trend of coil ESR is analyzed by changing the substrate thickness of the two layers at the same time. When the second layers of double-layer substrates are both aluminum plate, the value of coil ESR is calculated by COMSOL Multiphysics software when the first layer of substrate is Ferrite 1 and Ferrite 2 respectively, as shown in Figure 12.

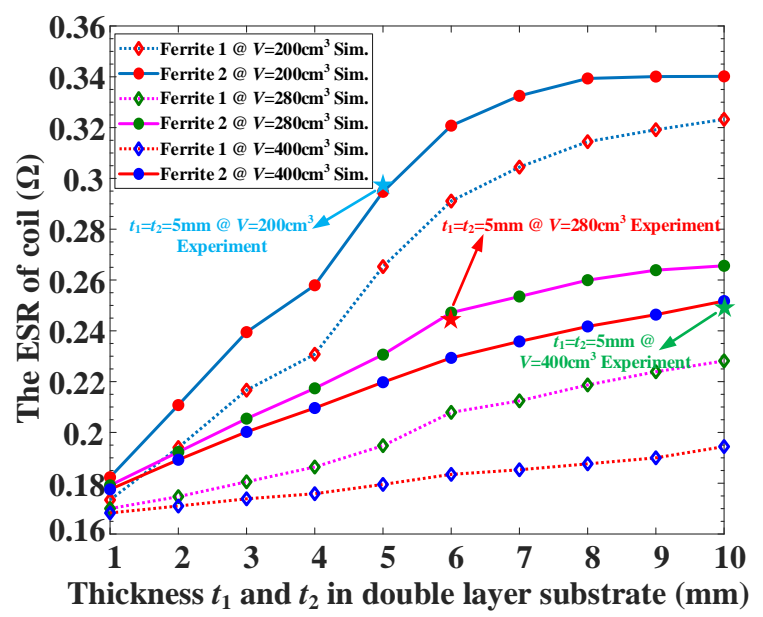

Figure 12. Coil ESR change curves when the weight of double-layer substrate material is constant.

As shown in Figure 12, at the same thickness of each layer of double-layer substrate, the larger the total volume of the substrate, the smaller coil ESR is. At the constant volume of double-layer substrate, coil ESR shall gradually increase with the increase of the thickness of each layer of substrate. When $V=200 \mathrm{~cm}^{3}$, the thickness of each layer of substrate greater than $5 \mathrm{~mm}$ shall be equivalent to the side length of each layer of substrate less than $20 \mathrm{~cm}$, that is, smaller than the diameter of the outer ring of the coil. According to the theory in Section 4.2, coil ESR shall demonstrate a sharp growth, which is consistent with the results in Figure 11. Hence, in the case of coil weight limitation, the thinner the thickness of double-layer substrate, the better. Of course, the conclusion is based on the volume reference value when $V=200 \mathrm{~cm}^{3}, V=280 \mathrm{~cm}^{3}$ and $V=400 \mathrm{~cm}^{3}$, and in the case of larger volume, the variation law of coil ESR with substrate thickness might be different. For WPT system, however, it is of significance to only discuss the case when the substrate is close to the coil size.

\section{Conclusions}

In view of the increase of coil ESR caused by substrate, based on theoretical modeling, simulation calculation, and experimental measurement, a systematic study on the variation rule of coil ESR with a single-layer substrate and double-layer substrate is carried out in this paper. According to the results, 
when the size of single-layer substrate is much larger than the coil size, the material with the smallest conductivity should be selected as the substrate. The thickness should be increased in case of large conductivity of the substrate, with an optimal value whereas. When the size of single-layer substrate is close to the diameter of the coil outer ring, coil ESR is proportional to the area of ferrite. However, with the increase of the area of aluminum plate, coil ESR increases first and then decreases. At the constant volume, the closer the side length of ferrite substrate is to the diameter of coil outer ring, the larger coil ESR is. Coil ESR value with aluminum plate reaches the minimum at the thickness of $5 \mathrm{~mm}$. For the double-layer substrate, when its size is far larger than the coil size, coil ESR is only subject to the thickness of ferrite substrate in the first layer, rather than that of aluminum plate in the second layer. When the size of substrate is close to the diameter of coil outer ring, coil ESR increases first and then decreases with the increase of area and finally tends to be constant. The peak value appears when the size is close to the diameter of coil inner ring. At the constant volume, coil ESR increases with the increase of the thickness of double-layer substrate. It is believed that the foresaid rules shall assist researchers' efficient selection of the thickness, area and volume of substrate, thus reducing the coil loss and realizing the optimal design of WPT system. Although this paper summarizes the influence of substrate with different size on the coil ESR, the variation law of the coil ESR needs further study when the excitation frequency of the coil, the distance between the substrate and the coil, and the shape of the substrate change.

Author Contributions: Conceptualization, J.L.; methodology, J.L., R.W., and M.Z.; software, J.L.; investigation, M.Z.; writing—original draft preparation, J.L.; writing—review and editing, L.T. and X.H. All authors have read and agreed to the published version of the manuscript.

Funding: This research was funded by the National Key Research and Development Project, grant number 2018YFB0106300.

Conflicts of Interest: The authors declare no conflict of interest.

\section{References}

1. Kurs, A.; Karalis, A.; Moffatt, R.; Joannopoulos, J.D.; Fisher, P.; Soljacic, M. Wireless power transfer via strongly coupled magnetic resonances. Science 2007, 317, 83-86. [CrossRef] [PubMed]

2. Huang, X.; Wang, W.; Tan, L. Technical progress and application development of magnetic coupling resonant wireless power transfer. Autom. Electr. Power Syst. 2017, 41, 2-14.

3. Liu, H.; Huang, X.; Tan, L.; Guo, J.; Wang, W.; Yan, C.; Xu, C. Dynamic Wireless Charging for Inspection Robots Based on Decentralized Energy Pickup Structure. IEEE Trans. Ind. Inform. 2018, 14, 1786-1797. [CrossRef]

4. Wen, F.; Chu, X.; Li, Q.; Gu, W. Compensation Parameters Optimization of Wireless Power Transfer for Electric Vehicles. Electronics 2020, 9, 789. [CrossRef]

5. Zhu, J.; Ban, Y.; Zhang, Y.; Yan, Z.; Xu, R.; Mi, C.C. Three-Coil Wireless Charging System for Metal-Cover Smartphone Applications. IEEE Trans. Power Electr. 2020, 35, 4847-4858. [CrossRef]

6. Nguyen, M.T.; Nguyen, C.V.; Truong, L.H.; Le, A.M.; Quyen, T.V.; Masaracchia, A.; Teague, K.A. Electromagnetic Field Based WPT Technologies for UAVs: A Comprehensive Survey. Electronics 2020, 9, 461. [CrossRef]

7. Huh, J.; Lee, W.; Cho, G.; Lee, B.; Rim, C. Characterization of Novel Inductive Power Transfer Systems for On-Line Electric Vehicles. In Proceedings of the 2011 Twenty-Sixth Annual IEEE Applied Power Electronics Conference and Exposition (APEC), Fort Worth, TX, USA, 6-11 March 2011; IEEE: Piscataway, NJ, USA, 2011; pp. 1975-1979.

8. Zhang, C.; Lin, D.; Tang, N.; Hui, S.Y.R. A Novel Electric Insulation String Structure With High-Voltage Insulation and Wireless Power Transfer Capabilities. IEEE Trans. Power Electr. 2018, 33, 87-96. [CrossRef]

9. Abou Houran, M.; Yang, X.; Chen, W. Magnetically Coupled Resonance WPT: Review of Compensation Topologies, Resonator Structures with Misalignment, and EMI Diagnostics. Electronics 2018, 7, 296. [CrossRef]

10. Joo, J.; Kwak, S.I.; Kwon, J.H.; Song, E. Simulation-Based System-Level Conducted Susceptibility Testing Method and Application to the Evaluation of Conducted-Noise Filters. Electronics 2019, 8, 908. [CrossRef] 
11. International Commission on Non-Ionizing Radiation Protection. Guidelines for limiting exposure to time-varying electric and magnetic fields (1 Hz to $100 \mathrm{kHz}$ ). Health Phys. 2010, 99, 818-836.

12. Yan, Z.; Li, Y.; Zhang, C.; Yang, Q. Influence Factors Analysis and Improvement Method on Efficiency of Wireless Power Transfer Via Coupled Magnetic Resonance. IEEE Trans. Magn. 2014, 50, 1-4.

13. Moon, J.; Shin, H.; Jeong, H.; Kim, S. Analysis of Receiver Circuit Efficiency for Wireless Power Transfer System According to Operating Frequency Variation. In Proceedings of the IEEE International Symposium on Consumer Electronics, Madrid, Spain, 24-26 June 2015; IEEE: Piscataway, NJ, USA, 2015.

14. Jeong, S.; Lin, T.; Tentzeris, M.M. Range-adaptive Impedance Matching of Wireless Power Transfer System Using a Machine Learning Strategy Based on Neural Networks. In Proceedings of the 2019 IEEE MTT-S International Microwave Symposium (IMS) 2019, Boston, MA, USA, 2-7 June 2019; IEEE: Piscataway, NJ, USA, 2019; pp. 1423-1425.

15. Zhao, L.; Thrimawithana, D.J.; Madawala, U.K.; Hu, A.P.; Mi, C.C. A Misalignment-Tolerant Series-Hybrid Wireless EV Charging System With Integrated Magnetics. IEEE Trans. Power Electr. 2019, 34, 1276-1285. [CrossRef]

16. Li, H.; Liu, Y.; Zhou, K.; He, Z.; Li, W.; Mai, R. Uniform Power IPT System With Three-Phase Transmitter and Bipolar Receiver for Dynamic Charging. IEEE Trans. Power Electr. 2019, 34, 2013-2017. [CrossRef]

17. Hurley, W.G.; Duffy, M.C. Calculation of self and mutual impedances in planar magnetic structures. IEEE Trans. Magn. 1995, 31, 2416-2422. [CrossRef]

18. Hurley, W.G.; Duffy, M.C. Calculation of self- and mutual impedances in planar sandwich inductors. IEEE Trans. Magn. 1997, 33, 2282-2290. [CrossRef]

19. Acero, J.; Alonso, R.; Burdio, J.M.; Barragan, L.A.; Puyal, D. Analytical equivalent impedance for a planar circular induction heating system. IEEE Trans. Magn. 2006, 42, 84-86. [CrossRef]

20. Acero, J.; Alonso, R.; Barragan, L.A.; Burdio, J.M. Modeling of Planar Spiral Inductors Between Two Multilayer Media for Induction Heating Applications. IEEE Trans. Magn. 2006, 42, 3719-3729. [CrossRef]

21. Su, Y.P.; Liu, X.; Hui, S.Y.R. Extended theory on the inductance calculation of planar spiral windings including the effect of double-layer electromagnetic shield. IEEE Trans. Power Electr. 2008, 23, 2052. [CrossRef]

22. Acero, J.; Alonso, R.; Burdio, J.M.; Barragan, L.A.; Puyal, D. Frequency-Dependent Resistance in Litz-Wire Planar Windings for Domestic Induction Heating Appliances. IEEE Trans. Power Electr. 2006, 21, 856-866. [CrossRef]

23. Lope, I.; Acero, J.; Carretero, C. Analysis and Optimization of the Efficiency of Induction Heating Applications with Litz-Wire Planar and Solenoidal Coils. IEEE Trans. Power Electr. 2016, 31, 5089-5101. [CrossRef]

24. Liu, J.; Deng, Q.; Czarkowski, D.; Kazimierczuk, M.K.; Zhou, H.; Hu, W. Frequency Optimization for Inductive Power Transfer Based on AC Resistance Evaluation in Litz-Wire Coil. IEEE Trans. Power Electr. 2019, 34, 2355-2363. [CrossRef]

25. Nguyen, M.H.; Fortin Blanchette, H. Optimizing AC Resistance of Solid PCB Winding. Electronics 2020, 9, 875. [CrossRef]

26. Tang, X.; Sullivan, C.R. Stranded wire with uninsulated strands as a low-cost alternative to litz wire. In Proceedings of the IEEE 34th Annual Conference on Power Electronics Specialist, 2003, PESC '03, Acapulco, Mexico, 15-19 June 2003; IEEE: Piscataway, NJ, USA, 2003; pp. 289-295.

27. Howe, G.W.O. The High-Frequency Resistance of Multiply-Stranded Insulated Wire. Proc. R. Soc. Lond. Ser. A Contain. Pap. Math. Phys. Character 1917, 93, 468-492.

(C) 2020 by the authors. Licensee MDPI, Basel, Switzerland. This article is an open access article distributed under the terms and conditions of the Creative Commons Attribution (CC BY) license (http://creativecommons.org/licenses/by/4.0/). 